\title{
Article \\ On Groups in Which Many Automorphisms Are Cyclic
}

\author{
Mattia Brescia ${ }^{1}\left(\mathbb{D}\right.$ and Alessio Russo ${ }^{2, *(D)}$ \\ 1 Dipartimento di Matematica e Applicazioni, Università di Napoli Federico II, 80138 Napoli, Italy; \\ mattia.brescia@unina.it \\ 2 Dipartimento di Matematica e Fisica, Università della Campania "Luigi Vanvitelli", 81100 Caserta, Italy \\ * Correspondence: alessio.russo@unicampania.it
}

check for updates

Citation: Brescia, M.; Russo, A. On Groups in Which Many

Automorphisms Are Cyclic.

Mathematics 2022, 10, 262. https://

doi.org/10.3390/math10020262

Academic Editors: Adolfo

Ballester-Bolinches and Ramón

Esteban-Romero

Received: 19 December 2021

Accepted: 13 January 2022

Published: 15 January 2022

Publisher's Note: MDPI stays neutral with regard to jurisdictional claims in published maps and institutional affiliations.

Copyright: (C) 2022 by the authors. Licensee MDPI, Basel, Switzerland. This article is an open access article distributed under the terms and conditions of the Creative Commons Attribution (CC BY) license (https:// creativecommons.org/licenses/by/ $4.0 /)$.

\begin{abstract}
Let $G$ be a group. An automorphism $\alpha$ of $G$ is said to be a cyclic automorphism if the subgroup $\left\langle x, x^{\alpha}\right\rangle$ is cyclic for every element $x$ of G. In [F. de Giovanni, M.L. Newell, A. Russo: On a class of normal endomorphisms of groups, J. Algebra and its Applications 13, (2014), 6pp] the authors proved that every cyclic automorphism is central, namely, that every cyclic automorphism acts trivially on the factor group $G / Z(G)$. In this paper, the class $F W$ of groups in which every element induces by conjugation a cyclic automorphism on a (normal) subgroup of finite index will be investigated.
\end{abstract}

Keywords: FC-groups; FW-groups; cyclic automorphisms; cyclicizer

MSC: 20E36; 20F24

\section{Introduction}

Let $G$ be a group. Following the work in [1], an automorphism $\alpha$ of $G$ is called a cyclic automorphism if the subgroup $\left\langle x, x^{\alpha}\right\rangle$ is cyclic for every element $x$ of $G$. Clearly, any power automorphism of $G$ (i.e., an automorphism which maps every subgroup onto itself) is cyclic; however, the multiplication by a rational number greater than 1 is a cyclic automorphism of the additive group of rational numbers which is not a power automorphism. Finally, it is easy to show that any cyclic automorphism of a periodic group is a power automorphism.

In [1], it was proved that any cyclic automorphism of a group $G$ is central, i.e., it acts trivially on the factor group $G / Z(G)$. Notice that this result is an extension to cyclic automorphisms of a renowned theorem by Cooper [2] for power automorphisms. It is not difficult to prove that the set $C A u t(G)$ of all cyclic automorphisms of $G$ forms a normal abelian subgroup of the automorphism group $A u t(G)$ of $G$. In [3], the structure of $C A u t(G)$ has been investigated in detail and some well-known properties of power automorphisms (see in [2]) has been extended to cyclic automorphisms. Moreover, the groups in which every automorphism is cyclic have been characterized there.

In the following, we will say that an element $g$ of a group $G$ induces by conjugation a weakly cyclic automorphism of $G$ if there exists a normal subgroup $W(g)$ of $G$ such that the index $|G: W(g)|$ is finite and the subgroup $\left\langle x, x^{g}\right\rangle$ is cyclic for each element $x$ of $W(g)$. Let $g_{1}$ and $g_{2}$ be elements of $G$ inducing weakly cyclic automorphisms and put $W=W\left(g_{1}\right) \cap W\left(g_{2}\right)$. If $x$ is an element of $W$, then $\left\langle x, x^{g_{1}}\right\rangle=\langle y\rangle$ for some $y \in W$, and so $\left\langle x, x^{g_{1}}\right\rangle$ is contained in the cyclic subgroup $\left\langle y, y^{g_{2}}\right\rangle$. It follows that $g_{1} g_{2}$ induces a weakly cyclic automorphism of $G$ and hence the set $F W(G)$ of all elements of $G$ inducing by conjugation weakly cyclic automorphisms of $G$ is a subgroup of $G$. Moreover, if $g$ is an element of $F W, x$ is an element of $W(g)$ and $y$ is an element of $G$, we have that $\left\langle x^{y^{-1}}, x y^{-1} g\right\rangle y$ is again a cyclic subgroup of $W(g)$, so that $F W(G)$ is a normal subgroup of $G$. We name this subgroup the $F W$-centre of $G$. A group which coincides with its $F W$-center will be called an FW-group. 
Recall that the cyclic norm $C(G)$ of a group $G$ is defined as the intersection of the normalizers of every maximal locally cyclic subgroup of $G$. By [3], Lemma 2.1, any cyclic automorphism of $G$ fixes all maximal locally cyclic subgroups of $G$. It follows that $C(G)$ coincides with the set of all elements of $G$ inducing cyclic automorphisms of $G$. In particular, $C(G)$ is a subgroup of $F W(G)$.

In the first part of the article, the class $\mathcal{F W}$ of groups in which every element induces by conjugation a weakly cyclic automorphism will be investigated. In particular, it will be proved that the class $\mathcal{F} \mathcal{W}$ coincides with the class $\mathcal{F P}$ recently studied by De Falco et al. [4]. Recall here that a group $G$ is said to be an FP-group if every element of $G$ induces by conjugation a power automorphism on some subgroup of finite index of $G$. Clearly, the groups with finitely many conjugacy classes (the so-called FC-groups) are FP-groups, while every $F P$-group is an $F W$-group. The consideration of the infinite dihedral group $D_{\infty}$ shows that there are $F P$-groups which are not $F C$-groups.

Let $G$ be a group and denote by $C y c(G)$ the set of all elements $x$ of $G$ such that $\langle x, y\rangle$ is cyclic for every $y$ in $G$. It is easy to show that $C y c(G)$ is a central, characteristic subgroup of $G$ called the cyclicizer of $G$ (see $[5,6])$. Clearly, $C y c(G)$ is locally cyclic and hence every automorphism of $G$ induces a cyclic automorphism on $C y c(G)$. In the last part of the article, groups with non-trivial cyclicizer will be investigated extending to the infinite case some results in [6-8]. In particular, it is shown that any torsion-free or primary generalized soluble group with non-trivial cyclicizer is an FW-group. Moreover, the well-known characterization of finite $p$-groups with only one subgroup of order $p$ (see, for instance, [9], 5.3.6) will be extended to locally finite groups. Finally, it is proved that the factor group $G / C y c(G)$ is finite if and only if $G$ has a finite covering of locally cyclic subgroups.

Most of our notation is standard and can be found in [10].

\section{FW-Groups}

Our first result is an easy remark concerning cyclic automorphisms of finite order.

Lemma 1. Let $G$ be a group. Every periodic cyclic automorphism of $G$ is a power automorphism.

Proof. Let $\alpha$ be a cyclic automorphism of $G$, let $g$ be an element of $G$, and consider a maximal locally cyclic subgroup $M$ of $G$ such that $g \in M$. As one can easily see that $M^{\alpha}=M$ (see, for instance, in [3], Lemma 2.1), then the normal closure $\langle x\rangle^{\langle\alpha\rangle}$ is locally cyclic and hence there exists an element $x$ of $G$ such that $\langle g\rangle^{\langle\alpha\rangle}=\langle x\rangle$. Clearly, $\langle x\rangle^{\langle\alpha\rangle}=\langle x\rangle$ and we may suppose that $g$ has infinite order. Therefore, $x^{\alpha}=x^{-1}$ and $g^{\alpha}=g^{-1}$. Thus, $\alpha$ induces a power automorphism on $G$.

Let $G$ be a group. A normal subgroup $W$ of $G$ is said to be weakly central if every element of $G$ induces by conjugation a cyclic automorphism of $W$. Clearly, if $G$ contains a weakly central subgroup of finite index, then $G$ is an $F W$-group.

Proposition 1. Let $G$ be a group. If $W$ is a weakly central subgroup of finite index of $G$, then every subgroup of $W$ is normal in $G$. In particular, $G$ is an FP-group.

Proof. First, assume that every inner automorphism of $G$ is cyclic. Then, $G$ coincides with its cyclic norm and hence every maximal locally cyclic subgroup of $G$ is normal. Let $g$ be an element of $G$ and consider a maximal locally cyclic subgroup $M$ containing $g$. As $G$ is an $F C$-group (see [3], Theorem 4.2), then the normal closure $\langle g\rangle^{G}$ of $g$ in $G$ is a finitely generated subgroup of $M$. Therefore, $\langle g\rangle$ is normal in $G$ and thus $G$ is a Dedekind group.

The above argument shows that $W$ is a Dedekind group. Since a cyclic automorphism of a periodic group is a power automorphism (see in [3], Lemma 2.3), we may suppose that $W$ is abelian. It follows that the factor group $G / C_{G}(W)$ is finite and hence every element $g$ of $G$ induces on $W$ a cyclic automorphism of finite order. The statement now follows from Lemma 1. 
Corollary 1. Let $G$ be a group all of whose inner automorphisms are cyclic automorphisms. Then $G$ is a Dedekind group.

Let $G$ be a group. We denote here with $F P(G)$ the $F P$-centre of $G$, namely the subgroup of all elements of $G$ inducing by conjugation power automorphisms on some subgroup of finite index of $G$. Clearly, $F P(G)$ is a subgroup of $F W(G)$.

Recall that a non-periodic group is said to be weak if it can be generated by its elements of infinite order, while it is said to be strong otherwise. In particular, all non-periodic abelian groups are weak.

Theorem 1. Let $G$ be a group. Then, FW-centre and FP-centre of $G$ coincide.

Proof. As the $F P$-centre of $G$ is a subgroup of $F W(G)$, we just have to show that every element of $G$ inducing a weakly cyclic automorphism of $G$ induces a weakly power automorphism of $G$. Therefore, let $g$ be an element of $F W(G)$ and let $W(g)$ be a normal subgroup of finite index of $G$ such that $g$ induces on $W(g)$ a cyclic automorphism. By Lemma 1, we may assume that $g$ induces an aperiodic automorphism on $W(g)$. Clearly, $g^{n} \in W(g)$ for some positive integer $n$ and $g^{n} \neq 1$. If $W(g)$ is weak, then $g$ acts universally on $W(g)$ (see [3], Theorem 3.5) and then $[W(g), g]=\{1\}$ as $g^{n}$ belongs to $W(g)$, so we may further assume that $W(g)$ is strong. If we let $W$ be the subgroup of $G$ generated by every element of infinite order of $G$, by Theorem 3.5 in [3], $g$ fixes $W$ and $G / W$ elementwise. Let now $x$ be an element of finite order of $W(g)$ and let $m$ be the order of $x$. As $\langle x\rangle$ and $\left\langle x^{g}\right\rangle$ are both subgroups of order $m$ of the cyclic group $\left\langle x^{g} x^{g}\right\rangle$, they coincide and this shows that $g$ acts as a power automorphism on every finite cyclic subgroup of $W(g)$. As $g$ centralizes every element of infinite order of $G$, it follows that $g$ induces a power automorphism on $W(g)$ and our thesis is proved.

Corollary 2. Let $G$ be a group. Then, $G$ is an FW-group if and only if $G$ is an FP-group.

Recall that a subgroup $X$ of a group $G$ is said to be pronormal if the subgroups $X$ and $X^{g}$ are conjugate in the subgroup $\left\langle X, X^{g}\right\rangle$ for all elements $g$ of $G$. As any subnormal and pronormal subgroup of a group is normal, it follows that a group all of whose subgroups are pronormal is a $\bar{T}$-group (i.e., a group in which normality is a transitive relation in every subgroup). However, the converse is false, as an example due to Kuzennyi and Subbotin [11] shows. We point out incidentally that in the universe of groups with no infinite simple sections the property $\bar{T}$ for a group $G$ is equivalent to saying that every subgroup of $G$ is weakly normal (see [12]). A tool which is useful to control pronormal subgroups of a group $G$ is the pronorm of $G$, which is defined as the set $P(G)$ of all elements $g$ of $G$ such that $X$ and $X^{g}$ are conjugate in $\left\langle X, X^{g}\right\rangle$ for any subgroup $X$ of $G$. The consideration of the alternating group $A_{5}$ shows that the pronorm of a group need not be in general a subgroup. On the other hand, the pronorm of a $\bar{T}$-group $G$ with no infinite simple sections is a subgroup of $G$ which coincides with the set $L(G)$ consisting of all elements $g \in G$ such that, if $H$ is a subgroup of $G$, then $g$ normalizes a subgroup of finite index of $H$ (see [13], Theorem 2.2). The last result of this section shows in particular that a $\bar{T}$-group $G$ with no infinite simple sections has all subgroups pronormal whenever $G$ belongs to the class $\mathcal{F} \mathcal{W}$.

Corollary 3. Let $G$ be a group. Then, $F W(G)$ is contained in $L(G)$. In particular, if $G$ is a $\bar{T}$-group with no infinite simple sections, $F W(G)$ is a subgroup of $P(G)$.

Proof. By Theorem 1, for every element $g$ of $F W(G)$ we may find a normal subgroup $W(g)$ of finite index of $G$ on which $g$ acts as a power automorphism. If we let $H$ be a subgroup of $G$, then the subgroup $H \cap W(g)$ of $W(g)$ is normalized by $g$, has finite index in $H$ and this proves our claim. 


\section{Groups with Non-Trivial Cyclicizer}

It is straightforward to see that a group with non-trivial cyclicizer is either torsion-free or periodic. Therefore, it is natural to inspect the cases in which the groups are either torsionfree or primary groups. As some arguments can be unified, in the following elements of infinite order will be said elements of order 0 and torsion-free groups will be called 0-groups.

Lemma 2. Let $G$ be a p-group where $p$ is a prime or 0 . If the cyclicizer $C y c(G)$ of $G$ is not trivial, then it coincides with the centre $Z(G)$ of $G$.

Proof. Assume for a contradiction that $C y c(G)$ is a proper subgroup of $Z(G)$. Then, we may find an element $x$ of $G$ and an element $y \in Z(G)$ such that $\langle x, y\rangle=\langle x\rangle \times\langle y\rangle$. Let now $c$ be a non-trivial element of $C y c(G)$. As the subgroups $\langle x, c\rangle$ and $\langle y, c\rangle$ are cyclic, there is a power of $c$ which belongs to $\langle x\rangle \cap\langle y\rangle=\{1\}$. It follows that $C y c(G)$ is periodic, so that also $G$ is periodic and hence the subgroups $\langle x, c\rangle$ and $\langle y, c\rangle$ have a unique subgroup of order $p$ for a prime $p$ dividing the order of, say, $\langle x, c\rangle$. In particular, the intersection $\langle x\rangle \cap\langle y\rangle$ is not trivial. This contradiction completes the proof.

The consideration of the direct product of a group of order 3 and a dihedral group of order 8 shows that there exists a (finite) group $G$ whose order is divided by only two primes and such that $\{1\} \neq C y c(G)<Z(G)$.

Let $A=\langle a\rangle$ be a cyclic group of order 4 , let $B$ be a group of type $2^{\infty}$ and let $b$ be an element of order 4 of $B$. Consider the semidirect product $H=A \ltimes B$ where $a$ acts as the inversion on $B$. Take $K=\left\langle a^{2} b^{2}\right\rangle$ and put $G=H / K$. Clearly, every finite non-abelian subgroup of $G$ is a generalized quaternion group. Therefore, in analogy with the locally dihedral 2-group $D_{2^{\infty}}$, we call $G$ a locally generalized quaternion group and we denote it with $Q_{2^{\infty}}$.

Here we give a first extension of Theorem 8 in [5].

Lemma 3. Let $G$ be a locally finite $p$-group for some prime $p$. Then, the cyclicizer of $G$ is not trivial if and only if

(1) G is locally cyclic or

(2) $G$ is isomorphic with a subgroup of $Q_{2^{\infty}}$.

In particular, if $G$ is finite and non-abelian, then $G$ is a generalized quaternion group.

Proof. Assume that the cyclicizer $C$ of $G$ contains a non-trivial element $c$ of order $p$. If $G$ is abelian, then Lemma 2 yields that $G$ coincides with its cyclicizer and then $G$ is locally cyclic. Assume thus that there exists a finite non-abelian subgroup $H$ of $G$ and let $x$ be an element of $\langle H, c\rangle$ of order $p$. As $\langle x, c\rangle$ is cyclic, one has that $x$ is a power of $c$, namely $\langle H, c\rangle$ contains a unique subgroup of order $p$. By a well-known characterization (see, for instance, [9], 5.3.6) we have that $\langle H, c\rangle$ is a generalized quaternion group. As this property holds for every finite subgroup of $G$ containing $\langle H, c\rangle$ and the set of finite subgroups of $G$ containing $\langle H, c\rangle$ is a direct system of $G$, we can clearly assume that $G$ is infinite. Therefore, it is possible to find in $G$ a subgroup $Q$ which is isomorphic with $Q_{2^{\infty}}$. Let $g$ be any element of $G$, let $P$ be the Prüfer 2-subgroup of $Q$ and let $y$ be an element of order $n>4$ of $P$. As $\langle g, y\rangle=\langle g, y, c\rangle$ is either a cyclic or a generalized quaternion group, we have in any case that $\langle y\rangle$ is normalized by $g$ and hence the whole $P$ is normalized by $g$. Moreover, $\langle g\rangle$ has non-trivial intersection with $P$, as both must contain $c$. Then, $g$ has to be contained in $Q$, otherwise $\langle g, Q\rangle$ would contain a direct product of two cyclic subgroups of order 2 . From this it immediately follows that $G$ is isomorphic with $Q_{2^{\infty}}$.

Let us prove the converse. If $G$ is locally cyclic the result is clear. On the other hand, take $G$ to be a subgroup of $Q_{2^{\infty}}$ which is not locally cyclic. Then, $G$ is not abelian, so that it is either the whole $Q_{2^{\infty}}$ or a generalized quaternion group. In both cases $Z(G)$ is the only subgroup of $G$ of order 2 and therefore it coincides with the cyclicizer of $G$, which is then non-trivial. 
This result gives a generalization to the locally finite case of the already quoted result about finite $p$-groups [9], 5.3.6.

Corollary 4. Let $p$ be a prime. A locally finite p-group $G$ contains exactly one subgroup of order $p$ if and only if it satisfies one of the following conditions:

(1) G is locally cyclic;

(2) $G$ is isomorphic with a generalized quaternion group;

(3) $G$ is isomorphic with $Q_{2}$.

In [7], it is proved that if $G$ is a torsion-free group such that cyclicizer $C y c(G)$ is not trivial, then $\operatorname{Cyc}(G)=Z(G)$ and if $Z(G)$ is divisible, then $G$ is locally cyclic. One may ask whether a torsion-free or a $p$-group with non-trivial cyclicizer is locally cyclic. In general, these questions can be answered in the negative because of two results by Olšanskii (see in [14], Theorem 31.4 and Theorem 31.5). On the other hand, our next result shows that for a wide class of generalized soluble groups the statement is true.

A group $G$ is said to be weakly radical if it contains an ascending (normal) series all of whose factors are either locally soluble or locally finite.

Theorem 2. Let $G$ be a locally weakly radical group such that $|\pi(G)| \leq 1$. Then, $G$ has non-trivial cyclicizer if and only if

(1) $G$ is locally cyclic or

(2) $G$ is isomorphic with a subgroup of $Q_{2}$.

Proof. Let $C$ be the cyclicizer of $G$. If $C \neq\{1\}$, it follows from Lemma 2 that $C=Z(G)$. Moreover, as already pointed out, $G$ is either torsion-free or periodic. By Lemma 3, we may also suppose that $G$ is torsion-free. Let $c$ be a non-trivial element of $C$. If $x$ is an element of $G$, then the subgroup $E=\langle x, c\rangle$ of $G$ is cyclic and hence there exists a positive integer $n$ such that $x^{n}$ belongs to $\langle c\rangle$. Thus the factor group $G / C$ is periodic and so even locally finite since $G$ is locally weakly radical. Now an easy application of a famous theorem by Schur (see, for instance, Corollary to Theorem 4.12 in [10]) shows that the commutator subgroup of $G$ is locally finite and hence $G$ is abelian. In particular, $G$ is locally cyclic.

The converse is an immediate consequence of Lemma 3.

Corollary 5. Let $G$ be a locally weakly radical group such that $|\pi(G)| \leq 1$. If $G$ has non-trivial cyclicizer, then it is an FW-group.

A straightforward application of Theorem 2 and of [9], 12.1.1 is the following.

Corollary 6. Let $G$ be a locally nilpotent group. Then $G$ has non-trivial cyclicizer if and only if either it is locally cyclic or $G$ is periodic and there is a prime number $p$ such that the $p$-component $G_{p}$ of $G$ either is locally cyclic or is isomorphic with a subgroup of $Q_{2}$.

A well-known result of Baer (see, for instance, in [10], Theorem 4.16) states that a group is central-by-finite if and only if it has a finite covering consisting of abelian subgroups. Furthermore, we have already quoted the theorem by Schur that ensures that a centralby-finite group is finite-by-abelian. In the following we rephrase these results replacing the centre $Z(G)$ of $G$ by the cyclicizer $C y c(G)$. Recall that a collection $\Sigma$ of subgroups of a group $G$ is said to be a covering of $G$ if each element of $G$ belongs to at least one subset in $\Sigma$.

Theorem 3. Let $G$ be a group and let $C$ be the cyclicizer of $G$. Then, the following hold:

(1) If $C$ has finite index in $G$, then $G$ is finite-by-(locally cyclic);

(2) The factor group $G / C$ is finite if and only if $G$ has a finite covering consisting of locally cyclic subgroups. 
Proof. (1) As $C \leq Z(G)$, then $G$ is central-by-finite and hence the commutator subgroup $G^{\prime}$ of $G$ is finite. Clearly, we may assume that $G$ is infinite, so that $C$ too is infinite and, by replacing $G$ with $G / G^{\prime}$, we may suppose that $G$ is abelian. Moreover, as $C$ is nontrivial, then $G$ is either torsion-free or periodic. In the former case, $G$ is locally cyclic by Proposition 2. Assume hence that $G$ is periodic. In this case, as we aim to show that $G$ is locally cyclic, we may also suppose that $G$ is a $p$-group for a prime $p$. However, $C$ is locally cyclic and hence of type $p^{\infty}$. It follows that $G$ can be decomposed as $G=C \times H$ where $H$ is a subgroup of $G$. If $c$ and $h$ are elements of order $p$ of $C$ and $H$, respectively, then the subgroup $\langle c, h\rangle$ is not cyclic. This contradiction shows that $H$ is trivial and hence $G=C$ is locally cyclic.

(2) First assume that the factor group $G / C$ is finite. Choose a (left) transversal to $C$ in $G$, say $\left\{x_{1}, \ldots, x_{n}\right\}$. Then, for any element $g$ of $G$, we can write $g=x_{i} c$ where $c$ is an element of $C$. Therefore, $g$ belongs to $\left\langle x_{i}, C\right\rangle$, which is locally cyclic, and $G$ is covered by the subgroups $\left\langle x_{i}, C\right\rangle$ with $i=1, \ldots, n$.

Conversely, assume that $G$ is covered by finitely many locally cyclic subgroups. Then by a result of Neumann (see in [10], Lemma 4.17) $G$ is covered by finitely many locally cyclic subgroups of finite index. Let $L$ be their intersection. Clearly, $L$ is contained in $C$ and $|G: L|$ is finite. It follows that $G / C$ is finite.

We remark that the cyclicizer of the direct product of $\mathbb{Z}_{2} \times \mathbb{Q}$ is trivial, so that the converse of point (1) of Theorem 3 is not true.

Author Contributions: Conceptualization, M.B. and A.R.; methodology, M.B. and A.R.; software, M.B. and A.R.; validation, M.B. and A.R.; formal analysis, M.B. and A.R.; investigation, M.B. and A.R.; resources, M.B. and A.R.; data curation, M.B. and A.R.; writing-original draft preparation, M.B. and A.R.; writing-review and editing, M.B. and A.R.; visualization, M.B. and A.R.; supervision, M.B. and A.R.; project administration, M.B. and A.R.; funding acquisition, M.B. and A.R. All authors have read and agreed to the published version of the manuscript.

Funding: This work was carried out within the "V:Valere 2019:Project: GoAL: VALERE: VAnviteLli pEr la RicErca".

Institutional Review Board Statement: Not applicable.

Informed Consent Statement: Not applicable.

Data Availability Statement: Not applicable.

Acknowledgments: The authors are supported by the "VALERE: VAnviteLli pEr la RicErca" project, by GNSAGA (INdAM) and are members of the non-profit association "Advances in Group Theory and Applications".

Conflicts of Interest: The authors declare no conflict of interest.

\section{References}

1. de Giovanni, F.; Newell, M.L.; Russo, A. On a class of normal endomorphisms of groups. J. Algebra Appl. 2014, 13, 6. [CrossRef]

2. Cooper, C.D.H. Power automorphisms of a group. Math. Z. 1968, 107, 335-356. [CrossRef]

3. Brescia, M.; Russo, A. On cyclic automorphisms of a group. J. Algebra Appl. 2021, 20, 13. [CrossRef]

4. De Falco, M.; Evans, M.; de Giovanni, F.; Musella C. Groups in which every element has a paracentralizer of finite index. Comm. Algebra 2020, 48, 2160-2166. [CrossRef]

5. O'Bryant, K.; Patrick, D.; Smithline, L.; Wepsic, E. Some facts about cycles and tidy groups. Rose-Hulman Institute of Technology, Indiana, USA, MS-TR 92-04. 1992. Available online: https://scholar.rose-hulman.edu/math_mstr/131/ (accessed on 18 December 2021).

6. Patrick, D.; Wepsic, E. Cyclicizers, centralizers and normalizers. Rose-Hulman Institute of Technology, Indiana, USA, MS-TR 91-05. 1991. Available online: https://scholar.rose-hulman.edu/math_mstr/74/ (accessed on 18 December 2021).

7. Abdollahi, A.; Hassanabadi, A.M. Noncyclic graph of a group. Comm. Algebra 2007, 35, 2057-2081. [CrossRef]

8. Abdollahi, A.; Hassanabadi, A.M. Noncyclic graph associated with a group. J. Algebra Appl. 2009, 8, 243-257. [CrossRef]

9. Robinson, D.J.S. A Course in the Theory of Groups, 2nd ed.; Springer: New York, NY, USA, 1995.

10. Robinson, D.J.S. Finiteness Conditions and Generalized Soluble Groups, 1st ed.; Springer: Berlin, Germany, 1972.

11. Kuzennyi, N.F.; Subbotin, I.Y. Groups in which all subgroups are pronormal. Ukrain. Math. J. 1987, 39, $251-254$. 
12. Russo, A. On groups in which normality is a transitive relation. Comm. Algebra 2012, 40, 3950-3954. [CrossRef]

13. Brescia, M.; Russo, A. On the pronorm of a group. Bull. Aust. Math. Soc. 2021, 104, 287-294. [CrossRef]

14. Olšanskii, A. Geometry of Defining Relations in Groups, 1st ed.; Kluwer Academic: Amsterdam, The Netherlands, 1991. 